\title{
Management preferences following radical inguinal orchidectomy for Stage I testicular seminoma in Australasia
}

\author{
G Hruby, ${ }^{1}$ R Choo, ${ }^{2}$ M Jackson, ${ }^{1}$ P Warde ${ }^{3}$ and H Sandler ${ }^{4}$ \\ ${ }^{1}$ Department of Radiation Oncology, Royal Prince Alfred Hospital, Sydney, New South Wales, Australia, ${ }^{2}$ Department of Radiation \\ Oncology, Toronto-Sunnybrook Regional Cancer Centre, ${ }^{3}$ Department of Radiation Oncology, Princess Margaret Hospital, Toronto, \\ Ontario, Canada and ${ }^{4}$ Department of Radiation Oncology, University of Michigan Medical Centre, Ann-Arbor, Michigan, USA
}

\section{SUMMARY}

A survey to evaluate the preferred patterns of management of Stage I seminoma was conducted during March 2001. The questionnaire was distributed by the Royal Australian and New Zealand College of Radiologists to all qualified radiation oncologists, 74 out of 170 responded. All performed a staging CT scan of the abdomen and pelvis. Thoracic imaging consisted of either chest X-ray (29\%) or chest CT (38\%) while $33 \%$ performed both. Fifty-four percent of radiation oncologists discussed surveillance with their patients but estimated that $5 \%$ or less would choose this option. The most commonly prescribed dose was 25 Gy in 15 or 20 fractions (79\%). Sixty-five percent of respondents treated the para-aortic (PA) nodes alone. Forty-two of 48 clinicians treating the PA field reported a change in practice after publication of the Medical Research Council study in 1999. Of these, 40 and $23 \%$ perform CT scans of the pelvis annually and every 6 months. Thirty-one percent did no follow-up CT scan. Compared to a similar survey from North America, we are more likely to use PA fields and less likely to discuss surveillance. As in the USA, and in contrast to Canada, few patients choose surveillance. There is no consensus regarding the frequency of follow-up scans in either North America or Australasia.

Key words: management; radiotherapy surveillance; testicular seminoma.

\section{INTRODUCTION}

Approximately $75 \%$ of patients with testicular seminoma present with Stage I disease. There are several management options for this stage of seminoma, including adjuvant radiotherapy (RT), surveillance or chemotherapy. The traditional management approach for Stage I pure seminoma has been radical inguinal orchidectomy followed by adjuvant external beam RT to the para-aortic and ipsilateral pelvic regions. With follow up consisting of clinical examination, serum tumour markers and chest X-ray (CXR), the long-term results are excellent with relapse-free rates of $94-98 \%$. With the use of salvage chemotherapy, long-term survival approaches $100 \% .^{1-3}$ While the acute toxicity of RT is low, there has been increasing concern with regards to potential long-term radiation-induced morbidity, including impaired spermatogenesis, chronic gastrointestinal complications and second malignancy. These concerns, together with the availability of improved imaging techniques and more effective salvage treatment for relapse, have led to the exploration of alternative management approaches and reduction of the intensity of RT. However, it is important to ensure that these changes do not compromise the current excellent survival outcomes.

A recent pooled analysis of four surveillance series comprising 638 patients, with a median follow up of 7 years, has reported a relapse rate of $18 \% .{ }^{4}$ The majority of recurrences (85-90\%) are limited to the para-aortic lymph nodes, and are successfully salvaged by RT. Although most first recurrences occur within 2 years of orchidectomy, recurrences have been

G Hruby FRANZCR; R Choo MD, FRCPC; $M$ Jackson FRANZCR; P Warde MB, MRCPI, FRCPC; $H$ Sandler MD.

Correspondence: George Hruby, Department of Radiation Oncology, Royal Prince Alfred Hospital, Missenden Road, Camperdown, NSW 2050,

Australia.Email: ghruby@email.cs.nsw.gov.au

Submitted 26 July 2001; accepted 4 April 2002. 
reported as late as 9 years from diagnosis. ${ }^{5-7}$ Thus, surveillance, which requires intensive follow up over a long period, depends upon both a motivated clinician and a compliant patient. Given these caveats, surveillance is a viable therapeutic alternative as it appears to provide a survival rate equivalent to that achieved with standard adjuvant RT.

Refinements to reduce the morbidity of RT have included reduction of the target volume. The Medical Research Council (MRC) trial comparing para-aortic and ipsilateral pelvic radiation or 'dog-leg' (DL) to a para-aortic strip alone (PA) in 478 patients reported no difference in disease-free or overall survival between the two arms. ${ }^{8}$ The acute toxicity, specifically haematologic, gonadal and gastrointestinal, was reduced in the PA arm. There were nine relapses in each arm, with four of those in the PA arm located in the pelvis. This was an equivalence trial, powered to detect a difference in pelvic relapse of greater than $3 \%$, and thus, small differences in outcomes might not have been detected.

There are reports of research utilizing one or two courses of single-agent chemotherapy instead of adjuvant RT. A German series of 93 patients treated with one cycle of carboplatin resulted in a relapse-free survival of $91 \%$, inferior to that achieved with adjuvant RT (94-98\%). ${ }^{9}$ By contrast, in a more recent publication from Vienna using two cycles of carboplatin in 107 men, there have been no relapses to date. ${ }^{10}$ Chemotherapy was generally well tolerated with minimal disruption to normal lifestyle. However, larger numbers and longer follow up are required to determine the late effects of this treatment.

With several treatment alternatives available for the management of Stage I seminoma of the testis, as well as variation with respect to radiotherapy treatment fields and prescribed doses, a survey was conducted among radiation oncologists to evaluate preferred management approaches to Stage I testicular seminoma.

\section{METHODS AND MATERIALS}

A survey was conducted between March and April 2001. The survey was sent to 174 practicing radiation oncologists registered with the Royal Australian and New Zealand College of Radiologists (RANZCR).

The Questionnaire consisted of three sections: (i) management approaches including the extent of baseline radiological investigations, whether or not the radiation oncologist discussed a surveillance option with their patients and, if so, the clinician's estimate of the proportion of patients opting for this strategy; (ii) a ranking of management options in the absence or presence of patient concern about fertility; and (iii) radiation oncologists were asked to nominate their preferred treatment volume and dose-fractionation schedule.

For proponents of the PA volume, a question concerning the impact of the MRC trial upon modifying their treatment volume was asked. Finally, again for oncologists treating the PA alone, a question regarding the frequency of CT scans for follow up of the pelvis, every 6 months, annually or not at all.

A similar survey was distributed in Canada and the USA between January and March 2001. ${ }^{11}$ Sections 1 and 3 were identical in both the North American and Australasian surveys, allowing comparison between the regions with respect to these parts of the survey. In North America, distribution of the survey differed to the Australasian survey. In Canada, the survey was sent to 93 radiation oncologists treating genitourinary tumours. In the USA, the survey was limited to the 36 Genitourinary Committee members of the Radiation Therapy Oncology Group (RTOG).

\section{RESULTS}

Seventy-four questionnaires were returned by the end of April. All responders were practicing radiation oncologists based in Australia, New Zealand and Singapore.

\section{Radiological investigations}

One person did not respond to any parts of the question regarding baseline investigations. Of 73 radiation oncologists who replied, all routinely performed a CT scan of the abdomen and pelvis. When evaluating the thorax, 21 ordered a CXR (29\%) and 28 ordered a chest CT scan alone (38\%), while 24 obtained both (33\%). Only one radiation oncologist requested a lymphangiogram (1.4\%). Three stated that they routinely performed ultrasound of the contralateral testis.

\section{Management options}

Just over half (54\%) of the respondents stated that they offered their patients the option of surveillance. Among those offering surveillance, they estimated that $5 \%$ or less (range $0-30 \%$ ) of the patients in their practice would choose this option (Table 1).

Radiation oncologists were asked to rank, in order of preference, three management options in the presence or absence of concern about fertility. The three management options were: (i) surveillance with reservation of RT or chemotherapy for relapse; (ii) postoperative adjuvant RT using either PA or DL field; and (iii) postoperative adjuvant single-agent chemotherapy.

In patients for whom fertility was a specific concern, $80 \%$ of radiation oncologists indicated that adjuvant radiotherapy would be their preferred management for that patient. In the absence of this concern, this increased to $96 \%$. Many of the replies did specifically state that sperm banking would be organized prior to $\mathrm{RT}$ if necessary. There was a corresponding reduction in the proportion of clinicians favouring surveillance as the preferred option for the two scenarios, falling from 18 to $3 \%$. Two radiation oncologists replied that they would favour adjuvant chemotherapy in the situation where fertility was an issue for the patient and in the absence of a fertility concern, this reduced to one.

\section{Adjuvant radiotherapy}

Seventy-five percent of Australasian radiation oncologists elected a para-aortic field alone as their treatment volume. 
Table 1. In your practice, do you offer the patient the option of surveillance, with salvage radiotherapy or chemotherapy reserved for relapse?

\begin{tabular}{lccc}
\hline & Yes & No & No response \\
\hline Number & 40 & 33 & 1 \\
Percentage & 54 & 45 & \\
\hline
\end{tabular}

If yes, what proportion of your patients opt for the surveillance? $14=0 \%, 4=$ no response, $7=10 \%, 5=5 \%, 3<10 \%, 3<5 \%, 1=20 \%$, $1=30 \%, 2=1 \%$. Median $5 \%$, range $0-30 \%$.

Thirty-four percent used a dog-leg and one described their volume as an 'amputated dog-leg', which treated the para-aortic and ipsilateral common iliac nodes. For the question regarding dose-fractionation schedules, this latter volume was included as a DL field.

Fifty-eight (79\%) respondents prescribed a dose of 25 Gy in either $15(41 \%)$ or $20(38 \%)$ fractions. Twelve percent used a dose of 30 Gy in either $15(7 \%)$ or $20(5 \%)$ fractions (Table 2).

Forty-two of 48 radiation oncologists had changed their treatment volume after the publication in 1999 of the MRC trial comparing para-aortic with para-aortic plus ipsilateral pelvic fields. Six had already been using the smaller volume and were regarded as not being influenced by this trial.

Those clinicians treating their patients with PA RT were asked to state the frequency of CT scanning to monitor the pelvis. As per Table 3, most (40\%) performed annual CT scans, $23 \%$ performed them every 6 months and $15(31 \%)$ did none.

\section{North American results}

Seventy-three of 79 Canadians and 24 of 36 US radiation oncologists returned the questionnaire.

Almost all radiation oncologists replied that they would order CXR (99\%) and CT scan of the abdomen and pelvis (100\%) as a baseline evaluation. Only one obtained a thoracic CT scan instead of CXR. Forty percent of North American radiation oncologists ordered both chest CT and CXR. Lymphangiogram was requested in $18 \%$. Both lymphangiogam and thoracic CT scan were more likely to be performed in the USA than Canada ( $25 \%$ vs. $16 \%$ ) and ( $45 \%$ vs. $38 \%)$, respectively.

Seventy-nine and $75 \%$ of Canadian and US respondents, respectively, stated that they would routinely recommend surveillance as a management option. When the option of surveillance was raised, the median estimated proportion of patients choosing it was 20\% in Canada (range $0-100$ ) and $7.5 \%$ in the US (range 0-50).

In Canada, the DL was more prevalent than the PA field (55 vs $42 \%$ ), whereas in the USA, 54\% used the PA field. Two North American radiation oncologists routinely used a 'modified dog-leg' that targeted the para-aortic and ipsilateral commmon iliac regions.

The most frequently used dose-fractionation schedule in
Table 2. Post-operative adjuvant radiotherapy for Stage I seminoma of testis?

\begin{tabular}{|c|c|c|c|c|}
\hline & Para-aortic & Dog-leg & Other & Total \\
\hline \multicolumn{5}{|c|}{ In this setting, what is your treatment volume? } \\
\hline$n$ & 48 & 25 & 1 & \\
\hline$\%$ & 65 & 34 & 1 & \\
\hline \multicolumn{5}{|c|}{ In this setting, what dose fraction schedule do you use? } \\
\hline $30 \mathrm{~Gy} / 20$ fractions & 2 & 2 & & $4(5 \%)$ \\
\hline 30Gy/15 fractions & 3 & 2 & & $5(7 \%)$ \\
\hline $25 \mathrm{~Gy} / 20$ fractions & 18 & 10 & & $28(38 \%)$ \\
\hline $25 G y / 15$ fractions & 22 & 8 & & $30(41 \%)$ \\
\hline Other & 3 & 4 & & $7(9 \%)$ \\
\hline
\end{tabular}

Table 3. If your treatment volume is the para-aortic region only, how often do you perform pelvic $\mathrm{CT}$ scan to assess pelvic failure after radiotherapy?

\begin{tabular}{lrr}
\hline & $n$ & $\%$ \\
\hline Annually & 19 & 40 \\
Every 6 months & 11 & 23 \\
Never & 15 & 31 \\
Other or no response & 3 & 6 \\
\hline
\end{tabular}

North America was 25 Gy in 20 fractions (68\%). In Canada, 73 and $21 \%$ prescribed 25 Gy in either 20 or 15 fractions, respectively. In the USA, $25 \mathrm{~Gy}$ in 20 fractions was most commonly used (54\%) with $21 \%$ prescribing $25-25.5$ Gy in 17 fractions.

Twenty-six percent of Canadians utilizing PA RT reported that they reduced their treatment volume from a dog-leg to paraaortic as a result of the MRC study whereas $38 \%$ did so in the USA.

Of those radiation oncologists using the PA field, 41 and $50 \%$ would perform a CT scan annually and every 6 months, respectively, for 3 years in order to monitor the pelvis. Only $9 \%$ did no follow-up CT scan.

\section{DISCUSSION}

There are several management approaches to Stage I testicular seminoma. This diversity partly reflects a paradigm shift from that of maximizing cure to one of minimizing treatmentrelated morbidity. Patient, treatment and tumour factors all play a role in the management decision, as do patterns of referral and the clinician's preferences and recommendations. This survey attempts to elicit management preferences among radiation oncologists belonging to the RANZCR.

As parts of the questionnaire are identical to a survey conducted in Canada and the USA during a similar period, certain practices can be compared and contrasted. However, it must be noted that the groups targeted in these three areas differed markedly. The Canadian Association of Radiation Oncologists has information on subsite specialization; therefore, 
all radiation oncologists treating genitourinary tumours could be identified, and by contacting individual provincial regional cancer centres, included in the survey. The survey was very selectively targeted in the USA where it was distributed only to the RTOG genitourinary committee members. By contrast, the survey distributed by the RANZCR might be over-inclusive as it targeted all practicing radiation oncologists affiliated with the College. Hence, the Australasian survey should be regarded as a study of management preferences rather than a definitive assessment of patterns of practice. At the opposite end of the spectrum, the US results reflect academic rather than community practice in that country. Finally, for a more comprehensive view, it might be important to include urologists and medical oncologists in future studies as they are involved in the patterns of referral and care of these patients.

\section{Radiological investigations}

With the exception of one radiation oncologist who did not answer any parts of the baseline investigation question, everyone performed a CT scan of the abdomen and pelvis. However, imaging of the thorax varied, with the majority (28) performing a CT scan, 24 ordering both CT and CXR, and 21 performing $\mathrm{CXR}$ alone. Only one radiation oncologist in Australasia routinely requested lymphangiography, reflecting both the invasive nature of the procedure and the fact that few radiologists continue to perform it given the availability of CT scanning. The ease of access to CT might also explain the use of CT for evaluating the thorax either instead of (38\%) or in addition to (33\%) CXR.

In North America, $40 \%$ of respondents ordered CT thorax in addition to CXR. A baseline CXR was performed by all except one clinician. The additional yield of thoracic $C T$ in the presence of a normal CXR is unknown. The use of lymphangiography continues to be much more common in North America than Australasia.

\section{Management options}

Many respondents ( $46 \%$ in the RANZCR) would not routinely offer the option of surveillance despite increasing evidence that surveillance with reservation of RT and/or chemotherapy for salvage appears not to compromise cure. There might be several reasons for this reluctance. Surveillance requires commitment by both the patient and their oncologist to intensive monitoring. It also requires prolonged follow up as late relapse (up to 9 years) is documented. Delay in the diagnosis of recurrence in the poorly compliant or highly mobile patient might compromise cure. Finally, surveillance carries both a psychological or emotional as well as an economic cost. The latter has been evaluated in two studies. ${ }^{12,13}$ In both, surveillance was more expensive than adjuvant dog-leg RT. Neither study incorporated the dollar cost of second malignancies into their calculations as this data is as yet unavailable for the surveillance series.
There was no significant difference in the proportion of radiation oncologists offering the option of surveillance between Canada and the USA. Both were more likely to offer surveillance than members of the RANZCR. However, when asked to estimate the proportion of patients choosing surveillance in their practice, there was a marked difference between the two countries. In Canada, radiation oncologists estimated that a median of $20 \%$ would opt for surveillance, whereas this estimate was only $7.5 \%$ in the USA, perhaps reflecting the fact that the Princess Margaret Hospital in Toronto, Canada, routinely offers the option of surveillance, and has a large experience with this approach. Locally, only $\leq 5 \%$ of patients were estimated to opt for surveillance, and just over half (54\%) of the clinicians stated that they routinely offered this option.

When a patient had specific fertility concerns, $80 \%$ of radiation oncologists indicated that adjuvant $\mathrm{RT}$ would be their favoured management. This increased to $96 \%$ in the absence of fertility preservation concerns. Thus, local radiation oncologists tended to favour adjuvant RT. Several respondents specifically stated that RT was their preferred alternative with the provision that sperm banking was performed prior to treatment. The survey did not examine the prevalence of cryopreservation prior to adjuvant treatment for this patient population. Somewhat surprisingly, two radiation oncologists favoured adjuvant chemotherapy in the situation where fertility was an issue for the patient. More data is needed in larger numbers of patients in order to fully evaluate the acute and late toxicities of chemotherapy. Controversy also exists as to the dose and type of agent, carboplatin has been shown to be inferior to cisplatin in the treatment of non-seminomatous germ-cell tumours. ${ }^{14,15}$ Finally, although the Austrian trial of two cycles of adjuvant carboplatin has no relapses amongst survivors, six patients died of other causes, a surprisingly high rate for a relatively young patient cohort.

\section{Postoperative adjuvant RT}

In Australasia, the MRC trial appears to have had a significant impact upon the treatment volume for postoperative RT. Almost two-thirds $(65 \%)$ of responders indicated that the PA field was their preferred volume, and of these, $88 \%$ stated that their practice had changed after publication of this trial. Twenty-six and $38 \%$ of Canadian and US clinicians changed their practice as a consequence of the MRC trial. With respect to treatment volumes, in Canada, a slight majority utilize the $\mathrm{DL}$ (58\%), while in the USA, 54\% favour the PA field.

Although the MRC trial comparing PA with DL radiotherapy was a large and well-conducted study, several issues remain unresolved. Specifically, given the risk of pelvic relapse after PA treatment, the issue of monitoring the pelvis has not been addressed. If follow-up pelvic $\mathrm{CT}$ is necessary and the MRC trial suggests it is, how often should it be done? In the MRC trial, CT scans of the abdomen and pelvis were done annually for 
3 years after RT in both arms. In the discussion section of the MRC paper, it was suggested that CT scanning every 6 months might be preferred for earlier detection of relapse as there were four pelvic relapses in the PA group. However, only two were isolated pelvic relapses, so the yield from scanning is very low. Philosophically, PA RT involves treating the most likely anatomical area of relapse with observation of the pelvis. Furthermore, the exact location of the four pelvic relapses (of which two were isolated) in the PA arm of the MRC trial were not given. If, for example, all the pelvic recurrences occurred in the common iliac lymph nodes, this raises the possibility that the optimal treatment field might in fact extend from T11 to the lower sacroiliac joints. Such a field would be associated with less acute toxicity than a DL, but might be associated with an even lower risk of pelvic relapse than PA alone, obviating the need for pelvic surveillance. ${ }^{16}$

The majority of Australasian radiation oncologists (79\%) used a total dose of $25 \mathrm{~Gy}$, delivered in either 15 (41\%) or 20 (38\%) fractions. For those treating to a total dose of $25 \mathrm{~Gy}$, a slightly larger proportion of clinicians used 20 fractions if treating a DL versus PA volume (56\% vs $45 \%$ ). Only $12 \%$ of radiation oncologists used a total dose of $30 \mathrm{~Gy}$. Thus, although the MRC trial has greatly affected local practice with respect to treatment volumes, a minority use the MRC dose/fractionation schedule (30 Gy in 15 fractions).

In North America, this effect is also evident with only $2 \%$ of North American radiation oncologists used 30 Gy in 15 fractions. The Canadians in particular have very uniform dose prescribing practices with $73 \%$ using 25 Gy in 20 fractions.

The frequency of pelvic CT scans after adjuvant PA RT varies between countries and individuals. In Australasia, $40 \%$ of radiation oncologists performed annual abdominal and pelvic CT scans, $23 \%$ organized scans for their patients every 6 months while $31 \%$ did not subsequently image the pelvis. By contrast, in North America, only $9 \%$ did not monitor the pelvis with the majority of clinicians divided between annual or twice-yearly CT scanning (41 and 50\%, respectively). Although the MRC trial recommended, but did not substantiate CT scans every 6 months, this is an area that requires further investigation.

\section{CONCLUSION}

There has been a trend towards using a smaller RT volume, strongly influenced by the published MRC trial. However, there is little consensus with respect to pelvic follow up after PA irradiation. Although $54 \%$ of radiation oncologists discuss the option of surveillance, few patients select this option.

Compared to North America, we are more likely to use PA fields and less likely to discuss surveillance as an option. A minority of our patients will opt for surveillance where this management alternative is discussed with the patient. Finally, although North American radiation oncologists are more likely than Australasians to perform CT scans of the pelvis after
PA treatment, there is no consensus regarding the frequency of these scans in any of these countries.

\section{ACKNOWLEDGEMENTS}

We would like to acknowledge the support of the RANZCR, those who distributed the survey and, finally, those clinicians who took the time to respond to the questionnaire.

\section{REFERENCES}

1. Epstein BE, Order SE, Zinreich ES. Staging, treatment and results in testicular seminoma: a 12 year report. Cancer 1990; 65: 405-11.

2. Schultz HP, von der Maase $H$, Rorth $M$. Testicular seminoma in Denmark 1976-80: Results of treatment. Acta Radiol Oncol 1984; 23: 263-70.

3. Thomas GM, Rider WD, Dembo AJ. Seminoma of the Testis. Results of Treatment and Patterns of Failure after Radiation Therapy. Int J Radiat Oncol Biol Phys 1982; 8: 165-74.

4. Warde $\mathrm{P}$, Specht $\mathrm{L}$, von der Maase $\mathrm{H}$ et al. Prognostic factors for relapse in Stage I seminoma managed by surveillance (Abstract). JUrol 1999; 161: 158.

5. Horwich A, Alsanjari N, A'Hern R, Nicholls J, Dearneley DP, Fisher $\mathrm{C}$. Surveillance following orchidectomy for Stage I testicular seminoma. Br J Cancer 1992; 65: 775-8.

6. von der Maase $\mathrm{H}$, Specht $\mathrm{L}$, Jacobsen $\mathrm{GK}$ et al. Surveillance following orchidectomy for Stage I seminoma of the testis. Eur $J$ Cancer 1993; 14:19 931-34.

7. Warde P, Gospodarowicz MK, Banerjee D et al. Prognostic factors for relapse in Stage I testicular seminoma treated with surveillance. J Urol 1997; 157: 1705-10.

8. Fossa SD, Horwich A, Russell MJ et al. Optimal planning target Volume for Stage I testicular seminoma: a Medical Research Council randomized trial. J Clin Oncol 1999; 17: 1146-54.

9. Dieckmann KP, Bruggeboes B, Pichlmeier U, Kuster J, Mullerleile U, Bartels H. Adjuvant treatment of clinical Stage I seminoma: Is a single course of carboplatin sufficient? Urology 2000;55: 102-6.

10. Reiter WJ, Brodwicz T, Alavi S et al. Twelve-year experience with two courses of adjuvant single-agent carboplatin therapy for clinical Stage I seminoma. J Clin Oncol2002; 19: 101-4.

11. Choo R, Sandler H, Warde P, Hruby G, DeBoer G. Survey of radiation oncologists: Practice patterns of the management of stage 1 seminoma of the testis in Canada and a selected group in the United States. Can J Urol 2002; 9: 1479-85.

12. Warde P, Gospodarowicz MK, Panzarella T et al. Long term outcome and cost in the management of Stage I testicular seminoma. Can J Urol 2000; 7: 967-72.

13. Sharda NN, Kinsella TJ, Ritter MA. Adjuvant radiation versus observation: a cost analysis of alternate management schemes in early-stage testicular seminoma. J Clin Oncol 1996; 14: 2933-9.

14. Bajorin DF, Sarosdy MF, Pfister DG et al. Randomized trial of etoposide and cisplatin versus etoposide and carboplatin in patients with good-risk germ cell tumours: a multi-institutional study. J Clin Oncol 1993; 11:598-606.

15. Horwich A, Sleijfer DT, Fossa SD et al. Randomized trial of bleomycin, etoposide and cisplatin compared with bleomycin, etoposide, and carboplatin in good prognosis metastatic nonseminomatous germ cell cancer: a multi-institutional Medical Research Council/ European Organization for Research and Treatment of Cancer trial. J Clin Oncol 1997; 15: 1844-52.

16. Thomas GM. Is optimal radiation for Stage I seminoma yet defined? J Clin Oncol 1999; 17: 3004-5. 